\title{
Improving the Efficiency of Administrative Decision-Making when Monitoring Reliability and Safety of Oil and Gas Equipment
}

\author{
Maria Zemenkova ${ }^{1, *}$, Viktor Shalay ${ }^{1}$, Yuri Zemenkov ${ }^{2}$, and Elena Kurushina ${ }^{1}$ \\ ${ }^{1}$ Industrial University of Tyumen, 625001 Volodarskogo str. 38, Tyumen, Russia \\ ${ }^{2}$ Omsk State Technical University, st. Mira 11, Omsk, Russia
}

\begin{abstract}
Methodology of rapid assessment of reliability index was developed based on system analysis of technological parameters. Within functioning of on-line monitoring system of reliability index of industrial facility this method allows to increase efficiency of making managerial decisions on technical and preventive maintenance. The technique is based on the analysis of technological parameters of operational modes of pipeline transport facilities registered by dispatcher controls. The created technique can be used by the operating, research, design institutes and oil and gas transport enterprises when declaring industrial safety. The received mathematical models allow federal services of supervision, the independent expert organizations to predict the development of reliability in the registered block of dispatching data either in real time mode, or taking into account the dynamics of service conditions of the object.
\end{abstract}

\section{Introduction}

The analysis of the existing legal and regulatory documentation, data bank of dispatch of oil and gas service companies suggests that a comprehensive assessment of the reliability of on-line mode requires a systematic model and universal mathematical models of the technical state of facilities with regard to the factors and parameters that change in long periods of operation and storage.

Modern database must contain all information to be registered by all monitoring tools for analysis, forecasting, decision-making in operation. The results of a retrospective analysis of objects indicate that the systems are not universal for oil and gas facilities, and require adaptation to a particular technical object, in accordance with the technological scheme, technical specifications and equipment models and other parameters that characterize a particular object [1-21].

Unfortunately, at present, the database for a comprehensive assessment of the reliability of on-line mode is not formed [6-10]. However, for the facility as a whole and its elements and subsystems at different levels certain operating parameters yi at fixed intervals are recorded. Diagnostic activities are performed in accordance with the schedule, the results of

\footnotetext{
* Corresponding author: muzemenkova@,mail.ru
} 
which determine the characteristics of the technical condition of the objects уід using experimental and computational methods. It should be noted that dispatching systems in oil-transport companies contain information about the actual operating parameters, which set common error standards defined by attorneys devices with a sufficiently small error $(0.4 \%)$. Therefore, as the reference information database we use all the parameters available for analysis and recorded by various monitoring tools: performance instrumentation; diagnostics; the results of tests and examinations, etc. At the same time in order to gain proper results the database must be uniform and recorded at regular intervals.

\section{Conditions of mathematical modeling}

Accurate values of operational and admissible parameters y are set for dispatching parameters in accordance with technological scheme. The basic condition of safe system of operation is the condition of absence of parameter outcome out of critical limits $y_{i} \in\left(y_{\min c r} ; \mathrm{y}_{\max c r}\right)$. At a deviation of parameters outside possible limits there is an exit of object out of operable state and inability to perform given functions, i.e. reliability. Loss of reliability can be associated with both factors depending on the operating conditions, and changing in the properties of the object in time, therefore it is necessary to evaluate conservation factors. Thus, failure can be seen as a physical and parametric. The problem of forecasting and reliability of the process $y(t)$ is reduced to the prediction of non-stationary random process and the determination of the probability of not outcoming of the random process beyond the tolerance values of $\mathrm{D}$ for a certain period of time tpred. Comprehensive analysis of the reliability of the system in accordance with the developed scheme involves an application of individual modeling technology of operation of the facility and the creation of models to assess the reliability of their parameters.

\section{Physical and mathematical model of reliability index}

Studying objects and solving the given tasks are related to the development of physical and mathematical models of reliability, reflecting the peculiarities of the phenomena and processes characteristic of pipeline transportation facilities. Under the term mathematical model hereinafter, we will understand the general formulation of indicators and a system of mathematical equations, in which it is possible to study the dynamics of changes in the reliability, getting information about the parameters and processes taking place.

We will specify the developed model of technical conditions with following terms and provisions:

1. The technical condition of the object is characterized by its set of output parameters y0, yx, ..., yn.

2. Characteristics of output signals (power, vibration, temperature, pressure, frequency, phase, duration of impulses etc.) or some system characteristics (productivity, efficiency etc.) are examined as output parameters.

3. Change of parameters is a random or deterministic process subjected to a certain distribution law.

4. Since the change of the parameters of technical objects (change in the vector $y=\left\{y_{0}, y_{1} \ldots y_{n}\right\}$ of operational characteristics) is influenced by many factors, it is fair to consider the evolution of parameters as a random function of time:

$$
y(\omega, \mathrm{t}), \mathrm{t} \in \mathrm{T}, \omega \in \Omega
$$


where $\Omega$ is the set of elementary events; $T$ is the interval of operation of the facility.

The main feature of the operation of the facility is non-stationarity of the processes (due to the regime change and irreversible aging and wear), and General model of the variation of parameters is a random function on a probability space $(\Omega, F, P)$ :

$$
y(t)=\left\{y_{j}(t)\right\}_{i=0}^{n}
$$

where $\mathrm{F}$ is algebra of subsets of sets $\Omega ; \mathrm{P}$ - probability measure.

5. If the targeted intervention is carried out in the system to achieve certain values of operational parameters yi, the process during this period can be considered as stationary deterministic with a given distribution function values:

$$
y(t)=F_{y}(t)
$$

6. The reliability index is determined by the function depending on two variables:

$$
K_{N}=F(y, t) .
$$

Thus, to build the mathematical model it is necessary to solve two problems:

1) determine the dependence $K_{N}=F(y)$

$$
\text { 2)define the dependency } K_{N}=F(t)
$$

The problem of optimal operational planning assessment and forecasting is the management: a priori random process $y(t)$ for uncontrolled objects; a posteriori random process $y^{P S}(t)$ with application of individual technical interventions for controlled objects.

In the control interval $T_{r}$ predictable process can be observed in the presence of some noise (measurement error) $\varepsilon(\mathrm{t})$. The overall task of the parameters drift forecasting can be formulated as a problem of prediction characteristics of a random process in the range of $\mathrm{Tr}$ according to the results of observations, distorted noise, which are obtained in the control range.

There are two components within creation of a model:

a) "observed" process:

$$
z(t), t \in T
$$

b) "unobservable" random process: $W(t), t \in T$ (statistically connected with $z(t), t \in T)$.

Let in a time point

$$
t \in T_{\mathrm{p}}, T_{p} \subset T
$$


where $\operatorname{Tr}$ - control interval (realization of "observed" process is known $z_{\omega}(t)$ ).

Changing of parameters is influenced by:

1. random factors;

2. deterministic processes.

Therefore, changes in the parameters are random or deterministic process, subject to a certain distribution law.

\section{Formulation of the problem}

Purpose of calculations:

1) evaluation of reliability index according to continuously changing technological parameter in real time;

2) definition of the function of parameter change over time;

3) forecasting of reliability index values in the prediction interval tprog.

Reliability assessment model in parameter is based on the following requirements of the established range of values $y_{i} \in\left(y_{\min c r} ; \mathrm{y}_{\max c r}\right)$

(fig. 1, 2):

$$
\begin{aligned}
& \text { 1) if } y=y_{\min \mathrm{Kp}}, y=y_{\max \mathrm{Kp}}, \mathrm{N}=0 \text {; } \\
& \text { 2) if } y=y_{\text {min cr }}+\frac{y_{\operatorname{mincr}}+y_{\operatorname{maxcr}}}{2}, \mathrm{~N}=1 \text {, }
\end{aligned}
$$

(in case the optimum technological parameter for object is not the middle of an interval $y_{i} \in\left(y_{\min c r} ; \mathrm{y}_{\max c r}\right)$, we admit under $\left.N=1, y=y_{\text {opt }}\right)$.

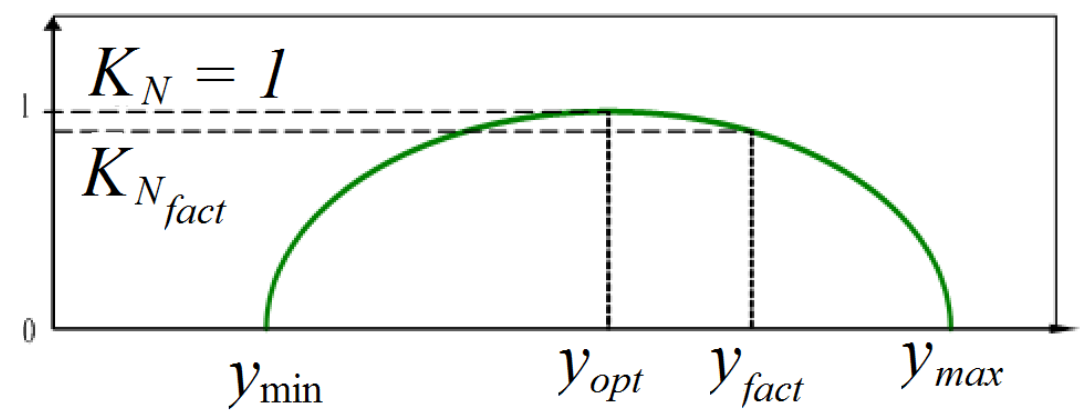

Fig. 1. Assessment scheme of reliability index in actual registered parameter. 
a)

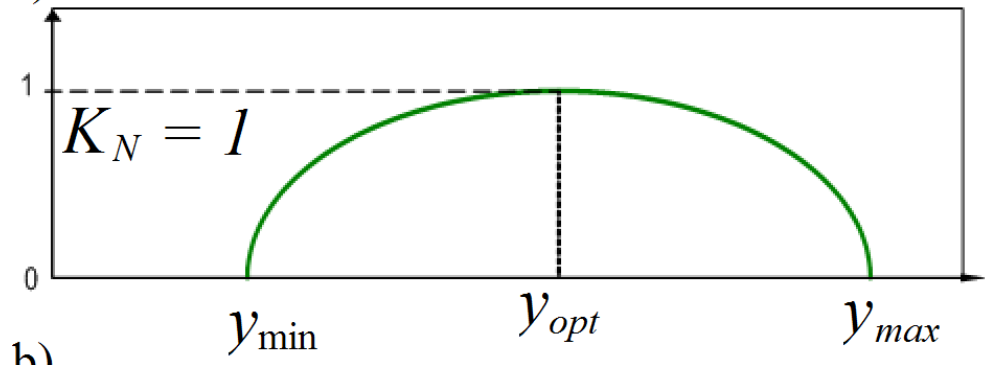

b)

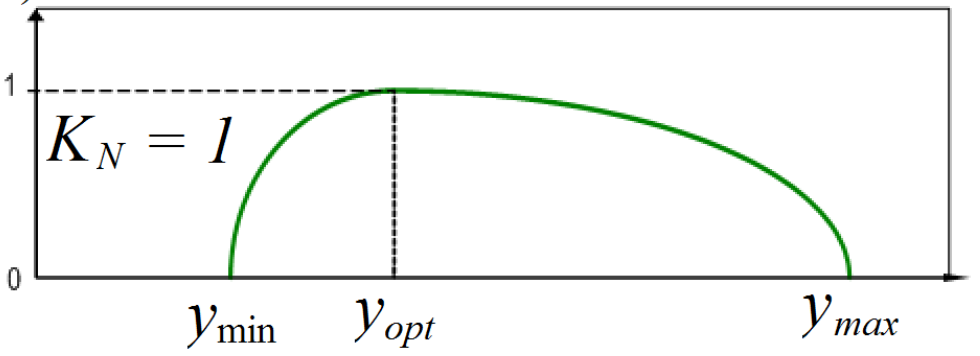

Fig.2. Dependence scheme in changing reliability index:

a) for symmetric and b) for asymmetric dependence of reliability function upon parameter change.

The advantage of the applied model is the continuous specification of distribution parameters in real time. Technique of realization of continuous monitoring of reliability is that at every moment of registration of the parameter calculation is performed on the reliability index of probability and statistical models.

Due to lack of a universal method it is necessary to develop the algorithm for determining distribution law of deviation of parameters, peculiarity of which is the ability to identify and clarify in real-time mode distribution parameters by statistical simulation modes.

Assume that for the solution of the given problem there is a number of operational registered parameters y of the volume n. Since the model works in real time, censored (incomplete) sampling is used for the calculations.

We will define the function of change of reliability depending on change of parameter of technical condition and the smallest volume of sampling for a distribution function assessment. According to classical methods of experiment planning at confidential probability 0.99 and the required accuracy up to $2 \%$ the minimum volume of selection makes $(55 \pm 19)$.

We will make statistical processing of the registered data by standard methods with the use of average arithmetic value.

As a result of data analysis we will also reveal the periods where function is determined or random.

Let the general function of distribution of parameter of technical condition in time be defined as the determined and casual constituent and has the following form (fig. 3):

$$
F(x)=y_{\text {det }}(t)+y_{\text {def }}(\mathrm{t})+\varepsilon
$$

Then, in addition to the standard "releases", we will exclude from the sampling the periods, where the system parameter level is purposefully maintained at the same level $y=$ const, i.e., factors determining the technical condition of the system are excluded. 


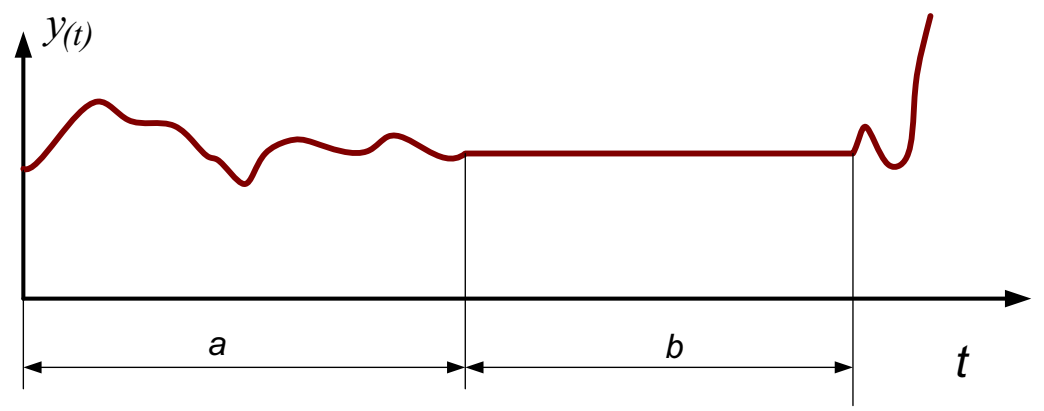

Fig. 3. Analysis of the function of the parameter over time.

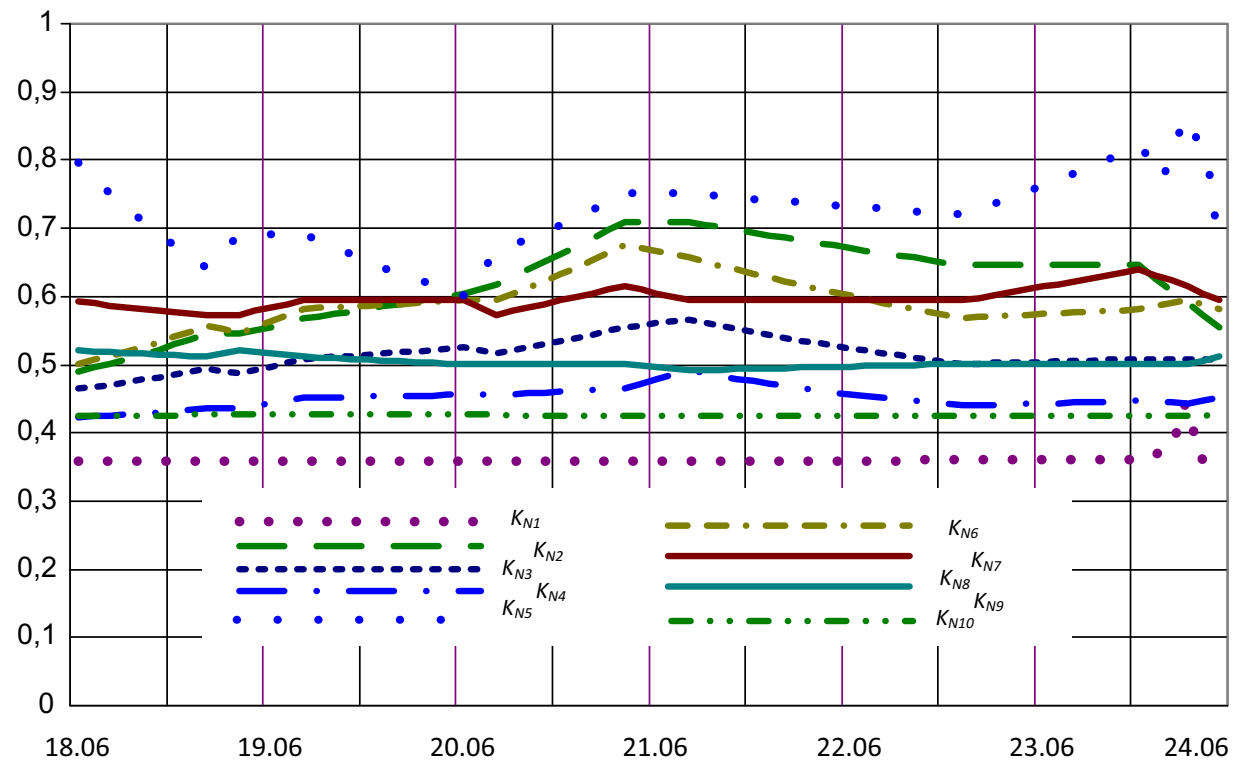

Fig.4. Monitoring results of reliability index of the pump of the retaining vertical pump at zero level.

As an example in fig. 4 it is shown that for one of object subsystems, for example, of the pump, various indicators of reliability can be received. The graph shows that in the analyzed period, the lowest rates are KN1, KN2, i.e. (according to interpretation) the lowest level of reliability have thrust bearing and the upper bearing of the pump due to high temperature and vibration and approximation of these parameters to the emergency values. Similar studies and calculations carried out by analytical and graphical dependencies at various levels of complexity of the system indicates that for the development of correct recommendations of elimination the causes of reduced reliability of pumping units the detailed and complex analysis of the modes and parameters during the entire period of operation of the equipment has to be carried out $[3,5,11]$.

To obtain adequate models of reliability it is very important to choose the type of distribution of failures or events corresponding to the actual, which largely depends on the accuracy of the results. To determine the type of distribution we use four known methods based on the application of: engineering data; graphical evaluation methods; nonparametric methods and evaluation criteria. 
The use of engineering data can prevent blunders at the stage of choosing a distribution. Here it is possible to estimate the kind of failure rate function and identify general information about the operated object. The use of graphical methods is justified on application of dispatching data or test data. Non-parametric methods are applied if it is not possible to evaluate satisfactorily the form of distribution, while the test time is equivalent to the required operating time of the equipment. Time as a factor, is possible to exclude and examine the signs of failure [15-21].

\section{Conclusion}

Thus, the developed technique allows carrying out an operational assessment of reliability index of an industrial facility and within monitoring increase efficiency of making administrative decisions on technical and preventive maintenance. The created technique can be used by research, design institutes and oil and gas transport enterprises when declaring industrial safety.

\section{References}

1. M. Zemenkova, PhD Thesis (TSOGU, Tyumen, 2007)

2. V. Kurushina, Yu. Zemenkov, WIT Transactions on Ecology and the Environment, 190(2), 881-888 (2014)

3. V. Antip'ev, A. Nevolin, Yu. Zemenkov, Neftyanoe Khozyaistvo - Oil Industry, 10, 46-48 (1981)

4. A. Bacherikov, M. Pashkov, Yu. Bogatenkov, Yu. Zemenkov, V. Shalay, G. Vasiliev, A. Prokhorov, S. Sidelnikov, S. Podorozhnikov, V. Kehl, Diagnosis in the maintenance of pipeline transportation (Nedra, St.Peterburg, 2007)

5. S. Dudin, Yu. Zemenkov, A. Shabarov, N. Saranchyn, Oil and Gas, 6, 63-68 (2010)

6. A. Shipovalov, Yu. Zemenkov, S. Toropov, et al., Aspects of technological reliability and economic efficiency of operation of underground storages of natural gas of Western Siberia (TSOGU, Tyumen, 2012)

7. A. Korolenok, Technological forecasting of capital repairs of the main gas pipelines (TSONIK GANG, Moscow, 1997)

8. Yu. Zemenkov, V. Shalay, M. Zemenkova, Procedia Engineering, 113, 254-258 (2015)

9. Yu. Zemenkov, V. Shalay, M. Zemenkova, Procedia Engineering, 113, 312-315 (2015)

10. M. Zemenkova, Yu. Zemenkov, A. Pimnev, V. Petryakov, Business magazine Neftegaz.RU, 11-12, 64-70 (2015)

11. S. Stepanov, N., Cheremisin, S., Sokolov, A., Altunin, A., Shabarov, ECMOR 2012 13th European Conference on the Mathematics of Oil Recovery (2012) DOI: 10.399/2214-4609.20143270

12. S. Toropov, Yu. Zemenkov, S. Podorozhnikov, S.Yu. Gas industry, 720(720), 95-98 (2015)

13. M. Zemenkova, L. Fayzulina, I. Seroshtanov, Gas industry, 720(720), 59-64 (2015)

14. A. Pimnev, M. Zemenkova, Pipeline transport: theory and practice, 5(51), 43-47 (2015)

15. A. Shabarov, A. Ibragimov, M. Zemenkova, S. Podorozhnikov, Gas industry, 12(715), 50-54 (2014)

16. O. Ivantsov, Reliability of building constructions of the main pipelines (Subsoil, Moscow, 1985)

17. I. Ryabinin, Reliability and safety of structural and difficult systems (Polyequipment, $\mathrm{SPb}, 2000)$ 
18. O. Abramov, A. Rosenbaum, Forecasting of a condition of technical systems (Science, Moscow, 1990)

19. V. Ostreykovsky, Theory of reliability (High school, Moscow, 2003)

20. E. Yasin, V. Berezin, K. Rashchepkin, Reliability of the main pipelines (Subsoil, Moscow, 1992)

21. I. Masur, O. Ivantsov, O. Moldavanov, Constructive reliability and ecological safety of pipelines (Subsoil, Moscow, 1990) 